\title{
Weighted protein residue networks based on joint recurrences between residues
}

\author{
Wael I. Karain ${ }^{1 *}$ and Nael I. Qaraeen ${ }^{2}$
}

\begin{abstract}
Background: Weighted and un-weighted protein residue networks can predict key functional residues in proteins based on the closeness centrality $C$ and betweenness centrality $B$ values for each residue. A static snapshot of the protein structure, and a cutoff distance, are used to define edges between the network nodes. In this work we apply the weighted network approach to study the $\beta$-Lactamase Inhibitory Protein (BLIP). Joint recurrences extracted from molecular dynamics MD trajectory positions of the protein residue carbon alpha atoms are used to define edge weights between nodes, and no cutoff distance is used. The results for $B$ and $C$ from our approach are compared with those extracted from an un-weighted network, and a weighted network that uses interatomic contacts to define edge weights between nodes, respectively.

Results: The joint recurrence weighted network approach performs well in pointing out key protein residues. Furthermore, it seems to emphasize residues with medium to high relative solvent accessibility that lie in loop regions between secondary structure elements of the protein.

Conclusions: Protein residue networks that use joint recurrences extracted from molecular dynamics simulations of a solvated protein perform well in pointing to hotspot residues and hotspot clusters. This approach uses no distance cutoff threshold, and does not exclude any interactions between the residues, including water-mediated interactions.
\end{abstract}

Keywords: Joint Recurrence, Protein Residue Networks, Solvent Dynamics, Molecular Dynamics

\section{Background}

The network paradigm has been used extensively to investigate protein structure and function [1-6]. The nodes in the network represent the protein residues. The edges between the nodes represent the strength of the residue interactions. The importance of a node can be predicted by calculating two parameters. The first is the closeness centrality $C$ which is defined as

$$
C_{n}=\frac{j-1}{\sum_{i \neq n} s d(i, n)}
$$

where $s d(i, n)$ is the shortest path between nodes $i$ and $n$, and $j$ is the number of nodes in the network. A node with a high $C$ plays a principal part in the transmission of information to all other residues in the network [7]. The second is the betweenness centrality $B$ which ranks

\footnotetext{
* Correspondence: wqaran@birzeit.edu

'Department of Physics, Birzeit University, Birzeit, Palestine

Full list of author information is available at the end of the article
}

the nodes according to the number of shortest paths passing through them between all the node pairs in the network. A node with a large $B$ value controls the flow of information in a network [8]. Nodes with large $B$ and $C$ values have been shown to lie in critical regions in proteins, and are usually binding free energy hotspots, or are located in the vicinity of hotspots $[9,2,10]$. Protein hotspot residues play a key role in protein-protein interactions. They can be detected experimentally using alanine mutagenesis [11].

Weighted and un-weighted protein residue interaction networks are based mostly on a static protein structure. The network nodes represent either the carbon alpha or beta atoms. In un-weighted networks, an edge of weight 1 is defined between nodes that are less than a cutoff distance apart. This distance is usually taken between 4 and $8.5 \AA$. In weighted networks, edge weights are based on inter-residue interaction strengths, which in turn depend on cutoff threshold distance. A typical example is a graph spectral method which was used to find central residues in proteins $[5,12,13]$. Edge weights were set 
equal to the reciprocal of the inter-node distance, given that the two residues satisfied a minimum interaction strength threshold subject to a distance cutoff threshold. To improve predictions, edge weights were also based on snapshots of the protein structure provided by calculation intensive molecular dynamics MD simulations. For example, two residues within a certain cutoff distance from each other in a certain percentage of MD trajectory frames were considered to be connected. Linear and nonlinear correlation coefficients between residues within a certain threshold cutoff distance, were also used to define edge weights $[14,15]$. Average values of non-bonded energy interactions between residues incorporated the chemical nature of residue interactions [16]. However, these methods ignored any long range residue-residue interactions, especially those by the surrounding water solvent [17]. This crucial coupling, facilitated by the forming and breaking of hydrogen bonds in the hydrogen bond network at the interface between the protein and the solvent, plays an important part in the dynamics of proteins [18-26]. It was taken into consideration by Bhattacharyya et al. who used an implicit solvent model to account for the effect of water on non-covalent energy interactions between two residues in a weighted protein residue interaction network [27]. This approach was successfully used to probe the allosteric mechanism in Pyrrolysyl-tRNA Synthetase. As a further refinement, we propose a method whereby edge weights can be defined in a way that includes all interactions, regardless of their nature, range, or value.

We have recently used the method of correlation of probability of recurrence CPR to find linear and nonlinear correlation coefficients between protein residue carbon alpha atoms [28]. This technique is based on the recurrence plot RP method [29]. Recurrence plots were introduced to visualize high dimensional phase space trajectories of dynamical systems using a 2-D map of the system's recurrences. The phase space trajectory of a given dynamical system is reconstructed using the method of time delays [30, 31]. Consequently, the recurrence plot locates recurring patterns in the dynamics of a system without making any previous assumptions about the nature of the dynamics. This technique can be especially useful when dealing with a-periodic and nonstationary systems, such as complex biological systems. To avoid the human bias inherent in studying graphical maps, the RP method was later developed to give quantitative results, and became known as recurrence quantification analysis RQA [32]. It has been used in many fields, including protein structure and dynamics [33]. The simultaneous recurrences by two or more systems, can also be detected using a multivariate version of the recurrence plot [34]. This joint recurrence plot JRP method can be used to quantify synchronization between two or more coupled dynamical systems. We hypothesize that if two residue carbon alpha atoms recur to their corresponding previous states at the same time instant, then they are interacting at that time instant. There is no need to know the nature of the interaction. By summing the total number of simultaneous recurrences over a given time period, an edge weight can be defined between the two atoms. A residue pair with a large number of joint recurrences is assumed to interact strongly. No threshold cutoff distance is needed to define the range of the interaction. However, each edge weight is divided by the geometric distance between the two residues [5]. This is justified because geometrically close residues with a given number of joint recurrences are expected to interact stronger than two residues that are far apart with the same number of joint recurrences. All interaction mechanisms, including those mediated by the solvent, are thus included.

The protein system we study in this work is the 165 residue $\beta$-Lactamase Inhibitory Protein BLIP. It is secreted by the soil bacterium Streptomyces clavuligerus. It inhibits $\beta$-lactam enzymes, which hydrolyze $\beta$-lactam antibiotics and nullify their effect [35-37]. It has been investigated extensively as a model system to understand the principles of affinity and specificity in proteinprotein interactions [38]. It has a large $\left(2636 \AA^{2}\right)$, concave, solvent exposed interaction interface consisting of an eight-stranded $\beta$-sheet. Numerous mutagenesis studies have pinpointed the hotspot residues that dominate its binding free energy with a number of $\beta$-Lactamase enzymes. These key residues are now known to be located in six independent hotspot clusters: C1:49; C2:74,142,143; C3:148,150,160; C4: 112,162; C5:36, 41, 50, 53; C6: 71,113 [39-45]. Most notably, residues Asp $49(\mathrm{C} 1)$ and Phe $142(\mathrm{C} 2)$ play a critical role in the binding process $[37,39]$. Residue $49(\mathrm{C} 1)$ also plays a dominant role in information flow between some of the hotspot clusters [46]. An ammonium ion binding site lies at residues 6 and 7 of BLIP. The sulfate ion binding site is at residues $12,13,14$, and 70 . This protein is suitable for our work on solvent-protein coupled dynamics due to the expected role the solvent pslay at such a large, extensively hydrated, interaction surface $[47,48]$.

In this work, a weighted network will be prepared for the BLIP protein. The residue carbon alpha atoms will be the nodes. Edge weights will be given by the cumulative number of joint recurrences of a residue pair over a time period of $10 \mathrm{~ns}$, divided by the distance between the two carbon atoms. The closeness and betweenness centrality values for each node will be calculated. For comparison, the BLIP protein will also be represented as an un-weighted network with a cutoff threshold distance of $7 \AA$, and a weighted network with edge weights based on inter-atomic contacts, calculated by the CSU program [49]. The results from these three networks will be compared. 


\section{Results and discussion}

We start by examining the solvent accessibility for residues with significant $B$ and $C$ values from the three networks for BLIP. Fig 1 shows a scatter plot of the $C \mathrm{z}$-score values versus the corresponding residue relative solvent accessibility RSA values. A significance z-score value of 1 is chosen arbitrarily to give a representative sample for each network. The average RSA values are $0.17,0.17$, and 0.37 , for the un-weighted, CSU-weighted, and JRP-weighted networks, respectively. Similarly, Fig. 2 shows a scatter plot of the $B \mathrm{z}$-score values larger than 1 versus the corresponding residue RSA values for the three networks. The average RSA values are $0.15,0.18$, and 0.40 , for the un-weighted, CSU-weighted, and JRP-weighted networks, respectively. The significant residues extracted from the JRP-weighted network have mostly medium and high RSA values. Amitai et al. [9] reported that the optimal parameters for protein active site prediction for a set consisting of 178 protein chains of enzymes with a total of 567 active sites were $C$ values $\geq 1.1$, and RSA values of $4.5-40 \%$. However, they also reported a general tendency for residues with high $C$ to be unexposed. Del Sol et al. [50] considered only residues with RSA $<20 \%$ in their work to identify key residues. To see if this is justified in the case of BLIP, a histogram of the RSA values for the experimentally determined BLIP hotspot residues is shown in Fig. 3. The majority of these residues has RSA values larger than $20 \%$, and can thus be regarded as being at least partially exposed [9]. In fact, the average RSA value for these hotspot residues is $31 \%$, ranging from 3 to $86 \%$. Thus, even though the conventional wisdom is to narrow the search for key residues among buried or partially exposed residues, one should not generalize this approach. Solvent exposed residues should be expected to play an important part in the control and flow of information inside the protein network.

Figures 4 and 5 show the standardized $B$ and $C$ values, respectively, for the residues represented as nodes in each of the three networks. The results are summarized in Tables 1, 2, and 3, for the un-weighted, CSU-weighted, and JRP-weighted networks, respectively. A z-score cutoff threshold of 2 is chosen arbitrarily for the $B$ values, and 1.5 for the $C$ values, respectively, to get a representative significant sample. Each table lists the significant nodes, and points out which ones are experimentally determined hotspots or ion binding sites. It also lists the first degree neighbors for each node, and points out if they are hotspot residues or ion binding sites as well.

Table 1 lists residues with significant $B$ and $C$ values detected by the un-weighted network. This list contains five experimentally determined hotspot residues: $53(\mathrm{C5})$, 71(C6), 74(C2), 112(C4), and 113(C6). The RSA values for these hotspots range from 0.13 to 0.28 . Five hotspot clusters, as well as the sulfate ion and the ammonium

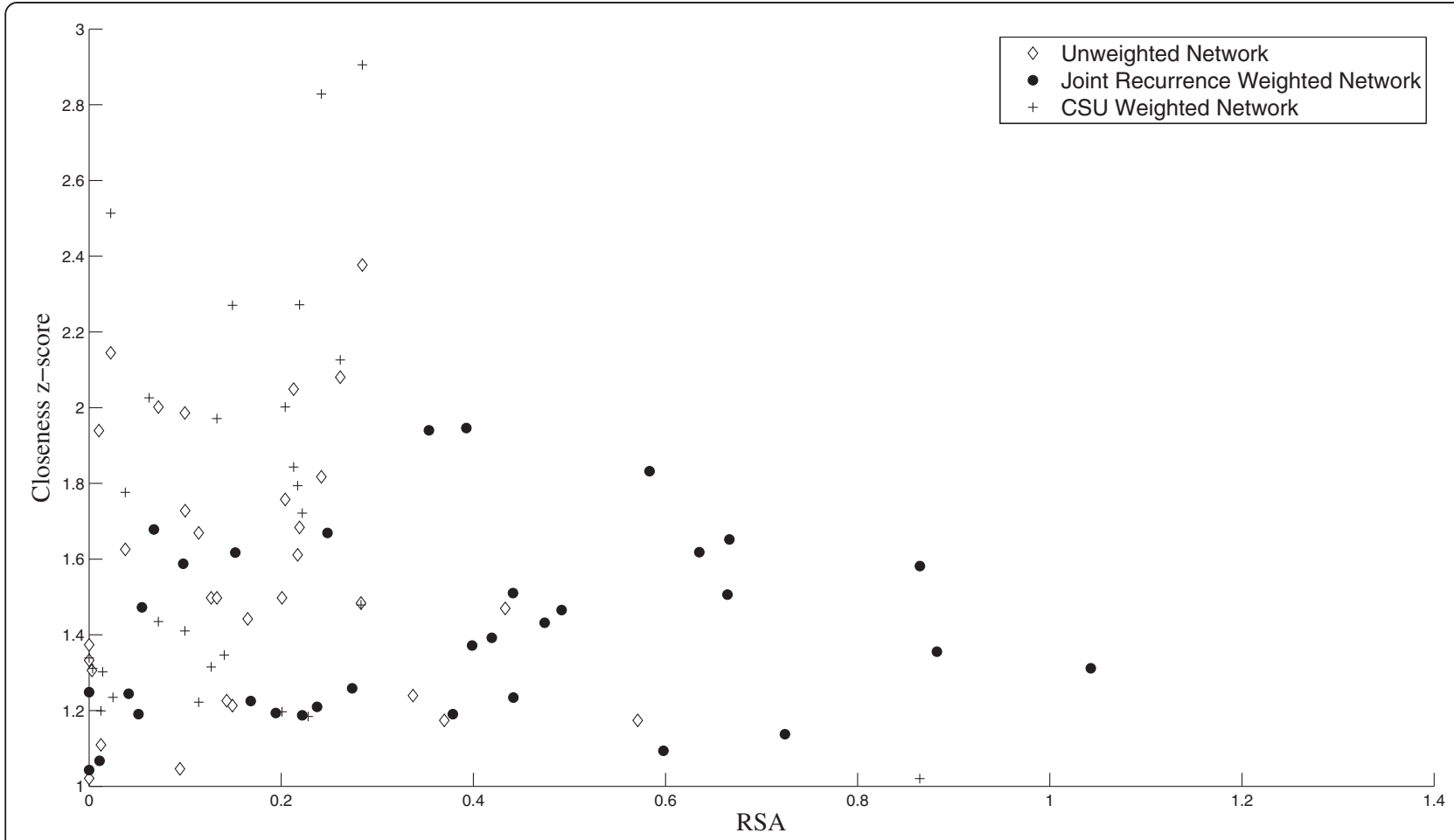

Fig. 1 The closeness centrality $C$ versus the relative solvent accessibility RSA. This figure shows the $C$ z-scores larger than 1 for the BLIP residues versus their RSA values for the un-weighted network with a $7 \AA$ cutoff threshold $(\diamond)$, the CSU weighted network (+), and the JRP weighted network $(\bullet)$ 


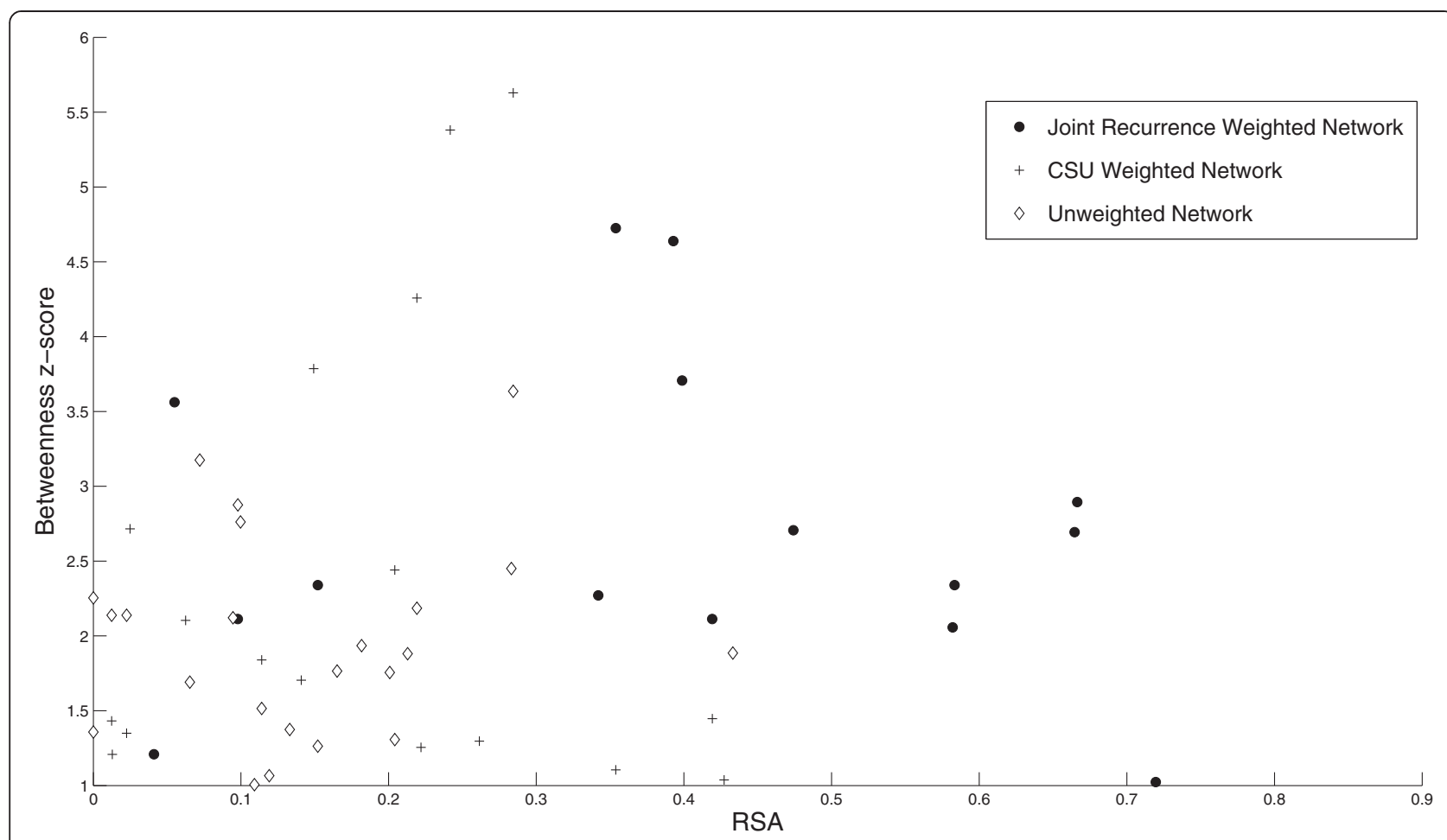

Fig. 2 The betweenness centrality $B$ versus RSA. This figure shows the $B$ z-scores larger than 1 for the BLIP residues versus their RSA values for the un-weighted network with a $7 \AA$ cutoff threshold ( () , the CSU weighted network (+), and the JRP weighted network $(\bullet)$

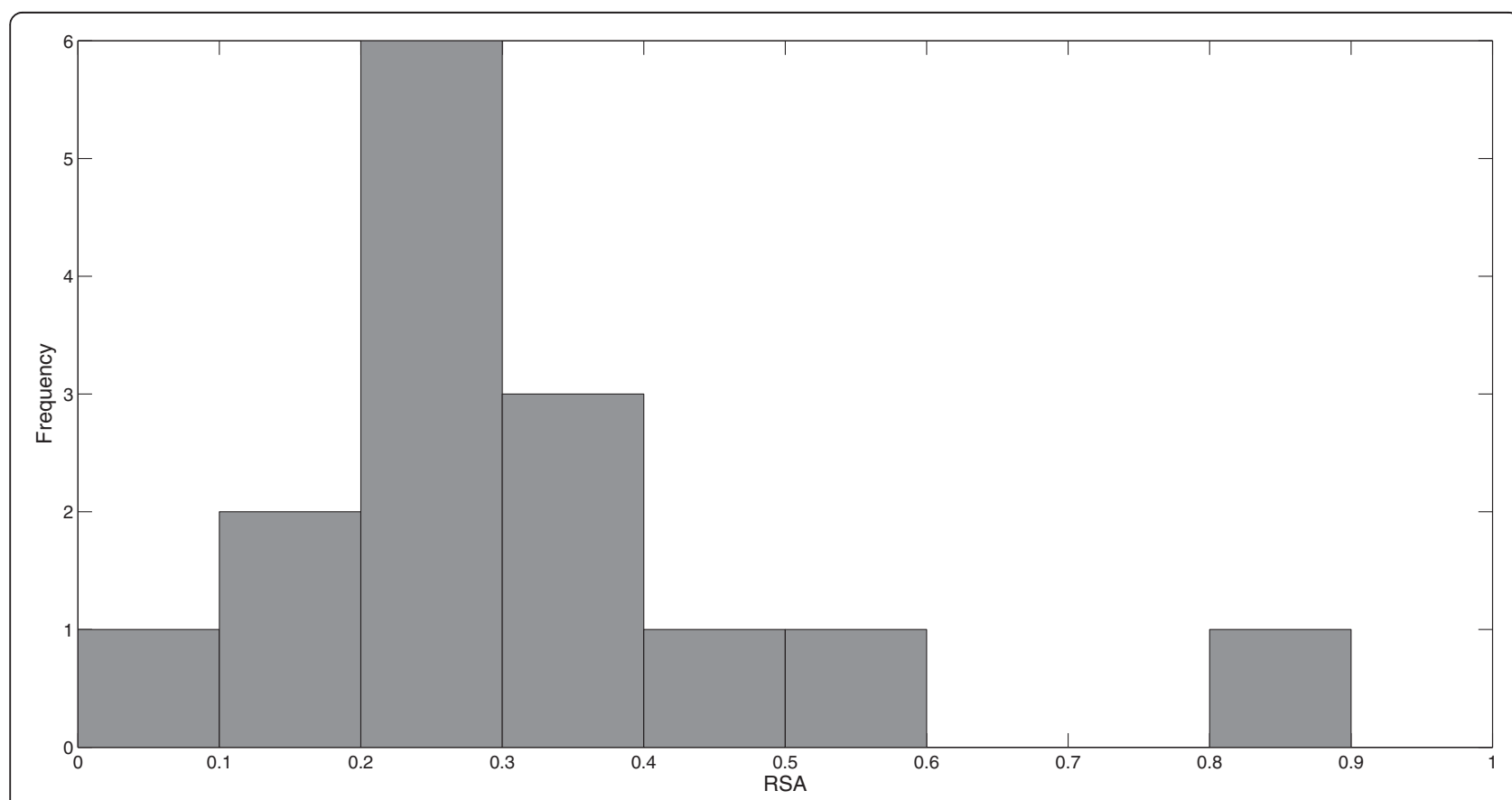

Fig. 3 The relative solvent accessibility RSA histogram for the BLIP hotspot residues. This figure shows the RSA value distribution for the experimentally determined hotspot residues in BLIP 

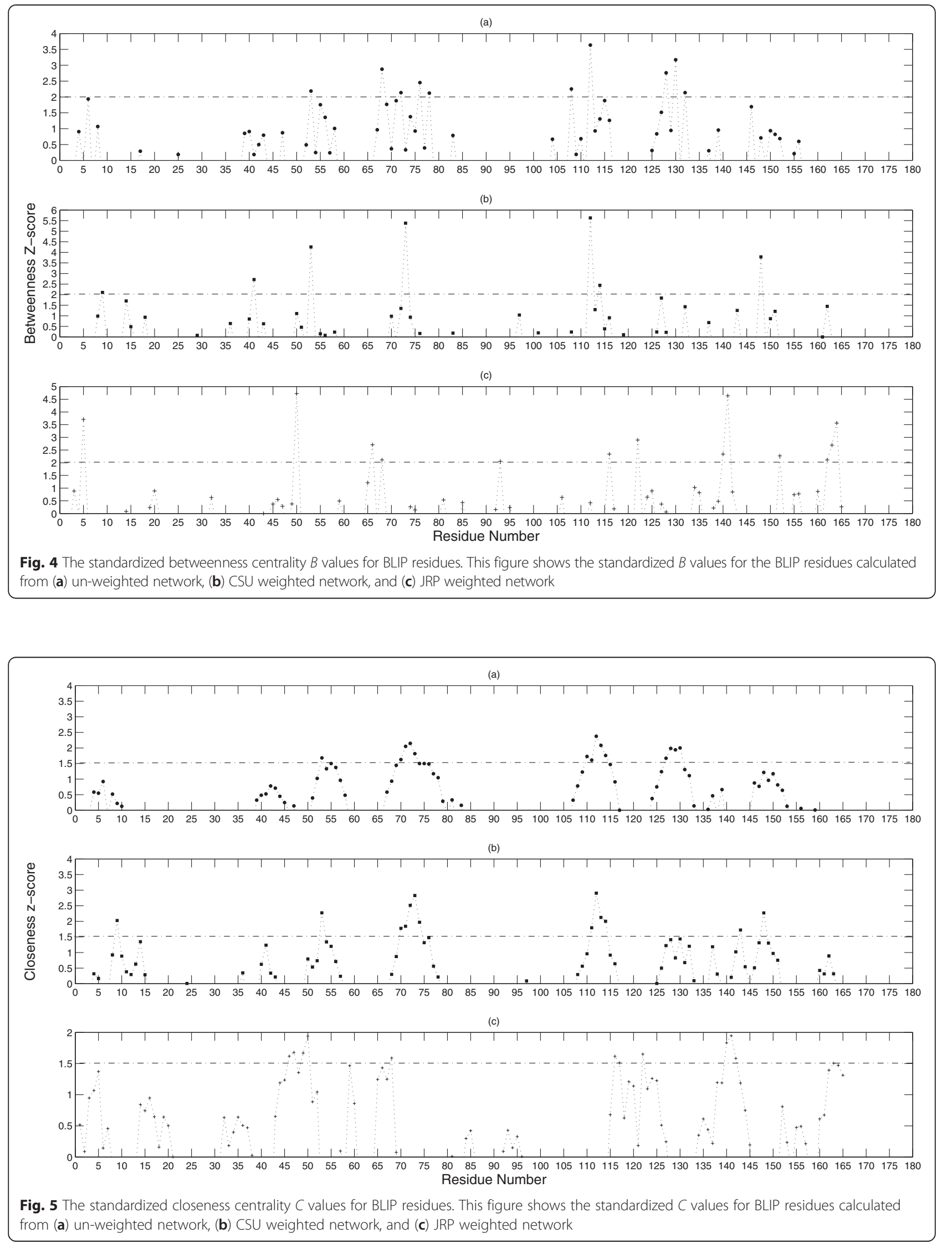
Table 1 Betweenness centrality and closeness centrality results for significant nodes in the un-weighted network

\begin{tabular}{|c|c|c|c|}
\hline Centrality & Residue \# & RSA & Significant neighbors \\
\hline Betweenness & $53(\mathrm{C} 5)$ & 0.22 & $41(C 5), 112(C 4)$ \\
\hline- & 68 & 0.10 & Sulfate ion binding site \\
\hline- & 72 & 0.02 & $53(\mathrm{C} 5), 71(\mathrm{C} 6), 74(\mathrm{C} 2), 112(\mathrm{C} 4)$ \\
\hline- & 76 & 0.03 & Ammonium ion binding site, $74(\mathrm{C} 2)$ \\
\hline- & 78 & 0.09 & - \\
\hline- & 108 & 0.00 & - \\
\hline- & $112(\mathrm{C} 4)$ & 0.28 & $53(\mathrm{C} 5), 71(\mathrm{C} 6), 148(\mathrm{C} 3)$ \\
\hline- & 128 & 0.10 & $112(\mathrm{C} 4), 150(\mathrm{C} 3)$ \\
\hline- & 130 & 0.07 & $112(\mathrm{C} 4), 148(\mathrm{C} 3)$ \\
\hline- & 132 & 0.01 & - \\
\hline Closeness & $53(C 5)$ & 0.22 & $41(\mathrm{C} 5), 112(\mathrm{C} 4)$ \\
\hline- & 55 & 0.20 & $36(C 5), 41(C 5), 53(C 5), 71(C 6)$, Sulfate ion binding site \\
\hline- & 70 (Sulfate ion binding site) & 0.04 & Sulfate ion binding site \\
\hline- & $71(\mathrm{C} 6)$ & 0.21 & 53(C5), Sulfate ion binding site, $112(\mathrm{C} 4), 113(\mathrm{C} 6)$ \\
\hline- & 72 & 0.02 & 53(C5), 71(C6), 74(C2), 112(C4) \\
\hline- & 73 & 0.24 & $53(\mathrm{C} 5), 74(\mathrm{C} 2), 112(\mathrm{C} 4), 148(\mathrm{C} 3)$ \\
\hline- & $74(\mathrm{C} 2)$ & 0.13 & $143(C 2)$ \\
\hline- & 75 & 0.13 & 53(C5), 74(C2), Ammonium ion binding site \\
\hline- & 110 & 0.10 & - \\
\hline- & 111 & 0.22 & 112(C4), Sulfate ion binding site \\
\hline- & $112(C 4)$ & 0.28 & $53(\mathrm{C} 5), 71(\mathrm{C} 6), 148(\mathrm{C} 3)$ \\
\hline- & $113(\mathrm{C} 6)$ & 0.26 & 112(C4), 71(C6), Sulfate ion binding site \\
\hline- & 114 & 0.20 & Sulfate ion binding site \\
\hline- & 127 & 0.11 & $150(C 3)$ \\
\hline- & 128 & 0.10 & $112(\mathrm{C} 4), 150(\mathrm{C} 3)$ \\
\hline- & 129 & 0.01 & $112(\mathrm{C} 4), 148(\mathrm{C} 3)$ \\
\hline- & 130 & 0.07 & $112(\mathrm{C} 4), 143(\mathrm{C} 2), 148(\mathrm{C} 3)$ \\
\hline
\end{tabular}

This table lists residues with betweenness centrality z-score values larger than 2 and closeness centrality values larger than 1.5. It also gives the relative solvent accessibility for each residue, and its first degree neighbors. The symbols between brackets denote hotspot clusters

ion binding sites, are among the first neighbors for residues in this list. The only cluster missing is $\mathrm{C} 1$, which consists of the key residue 49. Fig 6 shows the locations of these residues inside BLIP. In general, they are situated in the middle of the concave shaped active site.

Table 2 lists significant residues detected by the CSUweighted network. This list contains eight experimentally determined hotspot residues: 41(C5), 53(C5), 71(C6), 74(C2), 112(C4), 113(C6), 143(C2), and 148(C3). The RSA values for these hotspot residues range between 0.03 and 0.28 . Five hotspot clusters, as well as the sulfate ion and the ammonium ion binding sites, are among the first neighbors for the residues in this list. Cluster $\mathrm{C} 1$ is again not detected. Fig 7 shows the locations of these residues in BLIP. They are situated at the edge and the center of the active site.

Table 3 lists significant residues in the JRP-weighted network. This list contains four experimentally determined hotspot residues: 49(C1), 50(C5), 142(C2), and 162(C4). The RSA values for these hotspot residues range from 0.25 to 0.86 . Five hotspot clusters, including $\mathrm{C} 1(49)$, as well as the sulfate ion and the ammonium ion binding sites, are among the first neighbors for the residues in this list. Only cluster C6 $(71,113)$ is not detected. Most notably, the two "anchor" hotspot residues 49(C1) and 142(C2) are detected by the JRP-weighted network [51], while the two other networks fail to detect these important residues. Fig 8 shows the locations of these residues in BLIP. In clear contrast to the results in the un-weighted and CSU-weighted networks, these residues are located at the periphery of the protein interface surface.

Five BLIP residues are regarded as consensus hotspots for binding with the TEM-1, SHV-1, SME-1, and Bla1 enzymes: $36,41,49,53$, and 150 . The residues 148, 160 , and 162 are hotspots when BLIP binds all of the above enzymes with the exception of SHV-1 [40, 41]. The 
Table 2 Betweenness centrality and closeness centrality results for significant nodes in the CSU-weighted network

\begin{tabular}{|c|c|c|c|}
\hline Centrality & Residue & RSA & Significant neighbors \\
\hline Betweenness & 9 & 0.06 & Ammonium and Sulfate ion binding site, $71(\mathrm{C} 6)$ \\
\hline- & $41(C 5)$ & 0.03 & $36(C 5), 53(C 5)$ \\
\hline- & $53(C 5)$ & 0.22 & $41(C 5), 112(C 4)$ \\
\hline- & 73 & 0.24 & 53(C5), 74(C2), 112(C4), 143(C2) \\
\hline- & $112(\mathrm{C} 4)$ & 0.28 & $53(\mathrm{C} 5), 71(\mathrm{C} 6), 148(\mathrm{C} 3)$ \\
\hline- & 114 & 0.20 & Sulfate ion binding site \\
\hline- & 148(C3) & 0.15 & $112(\mathrm{C} 4), 143(\mathrm{C} 2), 150(\mathrm{C} 3), 160(\mathrm{C} 3), 162(\mathrm{C} 4)$ \\
\hline Closeness & 9 & 0.06 & 71(C6), Ammonium and Sulfate ion binding sites \\
\hline- & $53(C 5)$ & 0.22 & $41(C 5), 112(C 4)$ \\
\hline- & 70 (Sulfate ion binding site) & 0.04 & 112(C4), $71(\mathrm{C} 6)$, Sulfate ion binding site \\
\hline- & $71(\mathrm{C} 6)$ & 0.22 & 53(C5),112(C4), 113(C6), Sulfate ion binding site \\
\hline- & 72 & 0.02 & $53(\mathrm{C} 5), 71(\mathrm{C} 6), 74(\mathrm{C} 2), 112(\mathrm{C} 4)$ \\
\hline- & 73 & 0.24 & $53(\mathrm{C} 5), 74(\mathrm{C} 2), 112(\mathrm{C} 4), 148(\mathrm{C} 3)$ \\
\hline- & $74(\mathrm{C} 2)$ & 0.13 & 143(C2) \\
\hline- & 111 & 0.22 & 112(C4), Sulfate ion binding site \\
\hline- & $112(\mathrm{C} 4)$ & 0.28 & $53(\mathrm{C} 5), 71(\mathrm{C} 6), 148(\mathrm{C} 3)$ \\
\hline- & $113(\mathrm{C} 6)$ & 0.26 & 112(C4), 71(C6), sulfate ion binding site \\
\hline- & 114 & 0.20 & Sulfate ion binding site \\
\hline- & $143(C 2)$ & 0.22 & 74(C2), 142(C2), 148(C3) \\
\hline- & 148(C3) & 0.15 & $112(\mathrm{C} 4), 143(\mathrm{C} 3), 150(\mathrm{C} 3), 160(\mathrm{C} 3)$ \\
\hline
\end{tabular}

This table lists residues with betweenness centrality z-score values larger than 2 and closeness centrality values larger than 1.5 . It also gives the relative solvent accessibility for each residue, and its first degree neighbors. The symbols between brackets denote hotspot clusters

residues 73,74 , and 50 , are considered binding specificity determinants $[52,53]$. The un-weighted network detects residue $53(\mathrm{C} 5, \mathrm{RSA}=0.22)$ from the first group, and residue $74(\mathrm{C} 2, \mathrm{RSA}=0.13)$ from the third group. It does not detect any of the residues from the second group. The CSU-weighted network detects residues $41(\mathrm{C} 5, \mathrm{RSA}=0.03)$ and $53(\mathrm{C} 5, \mathrm{RSA}=0.22)$ from the first group, residue $148(\mathrm{C} 3, \mathrm{RSA}=0.15)$ from the second group, and residue $74(\mathrm{C} 2, \mathrm{RSA}=0.13)$ from the third group. The JRP-weighted network detects residue $49(\mathrm{C} 1, \mathrm{RSA}=0.25)$ from the first group, residue $162(\mathrm{C} 4, \mathrm{RSA}=0.42)$ from the second group, and residue $50(\mathrm{C} 5,0.35)$ from the third group. Again, the hotspots detected by the JRP-weighted network tend to have higher RSA values. This should be noteworthy since the significant hydration of the BLIP interface surface, has been proposed as a major factor in its "promiscuity", and ability to bind multiple enzymes $[37,48]$. It is also worth noting that a significant number of the residues with large $B$ and $C$ values extracted from the JRP weighted network, lie in the flexible loop regions between the secondary structure elements of the protein. It is also interesting that even though the hotspot residue 49 has a relatively low RSA value of 0.25 , it was not detected directly by the un-weighted or the CSU-weighted networks.

\section{Conclusions}

In this work we introduce the use of joint recurrences to represent interaction strengths between residue pairs in a weighted protein residue network. We also remove the cutoff distance condition used in defining edges, thus keeping long range interactions. We use betweenness and closeness centralities to detect key hotspot residues in the BLIP protein, which has a large, solvent exposed interaction surface. Our JRP-weighted network results compare well with those extracted from an un-weighted network with a $7 \AA$ cutoff distance, and a weighted network with the edge weights defined by the number of the inter-atomic contacts between residues. While the CSU-weighted network detected more hotspots, the JRP-weighted network was able to detect the "anchor" residues $49(\mathrm{C} 1)$ and $142(\mathrm{C} 2)$. Our results indicate that residues with significant $B$ and $C$ values are either themselves, or are in contact with, hotspot residues or ion binding sites. Our approach also favors residues that lie in loop regions between secondary structure elements, and that are partially or highly exposed to the surrounding solvent. This seems more appropriate for this "promiscuous" protein, which has experimentally determined hotspot residues that tend to be either partially or highly 
Table 3 Betweenness centrality and closeness centrality results for significant nodes in the JRP-weighted network

\begin{tabular}{|c|c|c|c|}
\hline Centrality & Residue & RSA & Significant neighbors \\
\hline Betweenness & 5 & 0.40 & Ammonium ion binding site \\
\hline- & $50(C 5)$ & 0.35 & $36(C 5), 41(C 5), 49(C 1)$ \\
\hline- & 66 & 0.47 & - \\
\hline- & 68 & 0.10 & Sulfate ion binding site \\
\hline- & 93 & 0.58 & - \\
\hline- & 116 & 0.15 & Sulfate ion binding site \\
\hline- & 122 & 0.67 & - \\
\hline - & 140 & 0.58 & $74(C 2)$ \\
\hline- & 141 & 0.39 & $74(C 2), 142(C 2), 143(C 2)$ \\
\hline- & 152 & 0.34 & - \\
\hline- & $162(C 4)$ & 0.42 & 148(C3), 150(C3), 160(C3) \\
\hline- & 163 & 0.67 & $142(\mathrm{C} 2)$ \\
\hline- & 164 & 0.05 & - \\
\hline Closeness & 46 & 0.64 & $50(C 5)$ \\
\hline- & 47 & 0.07 & $49(C 1), 50(C 5), 74(C 2)$ \\
\hline- & $49(\mathrm{C} 1)$ & 0.25 & $50(C 5)$ \\
\hline- & $50(C 5)$ & 0.35 & $36(C 5), 41(C 5), 49(C 1), 53(C 5)$ \\
\hline- & 68 & 0.10 & Sulfate ion binding site \\
\hline- & 116 & 0.15 & Sulfate ion binding site \\
\hline- & 117 & 0.44 & - \\
\hline \multirow[t]{5}{*}{ - } & 122 & 0.67 & - \\
\hline & 140 & 0.58 & $74(C 2)$ \\
\hline & 141 & 0.39 & $74(C 2), 142(C 2), 143(C 2)$ \\
\hline & $142(C 2)$ & 0.86 & $143(C 2)$ \\
\hline & 163 & 0.67 & 142(C2), 162(C4) \\
\hline
\end{tabular}

This table lists residues with betweenness centrality z-score values larger than 2 and closeness centrality values larger than 1.5 . It also gives the relative solvent accessibility for each residue, and its first degree neighbors. The symbols between brackets denote hotspot clusters

exposed to the solvent, and which presents a large interaction surface to the solvent. In general, protein networks should not neglect any interaction mechanisms.

\section{Methods}

The starting point in reconstructing the phase space trajectory $X(t)$ of a given dynamical system, is a scalar time series of one of the system's measured or calculated observables $Z(t)$, where $Z=\left(Z_{1}, Z_{2}, \ldots, Z_{N}\right)$ consists of evenly spaced single values 1 to $\mathrm{N}$. The reconstructed trajectory reflects the underlying dynamics of the original system. Each point in the reconstructed phase trajectory represents a possible state for that system, and is given by a vector

$$
\vec{X}_{i}=\left(Z_{i},, Z_{i+d}, \ldots, Z_{i+(k-1) d}\right) \quad k=1, \ldots, m
$$

where $d$ is the delay parameter, and $m$ is the embedding dimension. The delay parameter $d$ is estimated by

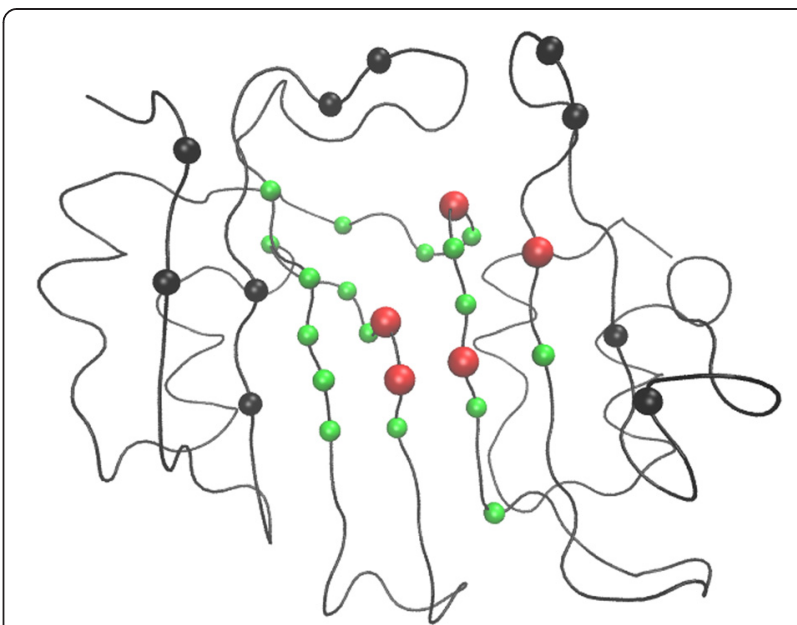

Fig. 6 The locations of the significant un-weighted network residues inside BLIP. This figure shows the locations of significant BLIP residues with $B z$-score values larger than 2 , and $C$ z-score values larger than 1.5 . The black colored spheres are experimentally determined hotspots. The red colored spheres are experimentally determined hotspots detected by this network. The green colored spheres are significant residues determined by this network

finding the first minimum in the mutual information function. This function can detect linear and nonlinear correlations between the elements of the scalar time series [54]. A suitable delay parameter should give the least amount of shared information between the vector components, while at the same time keeping them related. The embedding dimension $m$ is then estimated using the method of false nearest neighbors [55]. Choosing the correct embedding

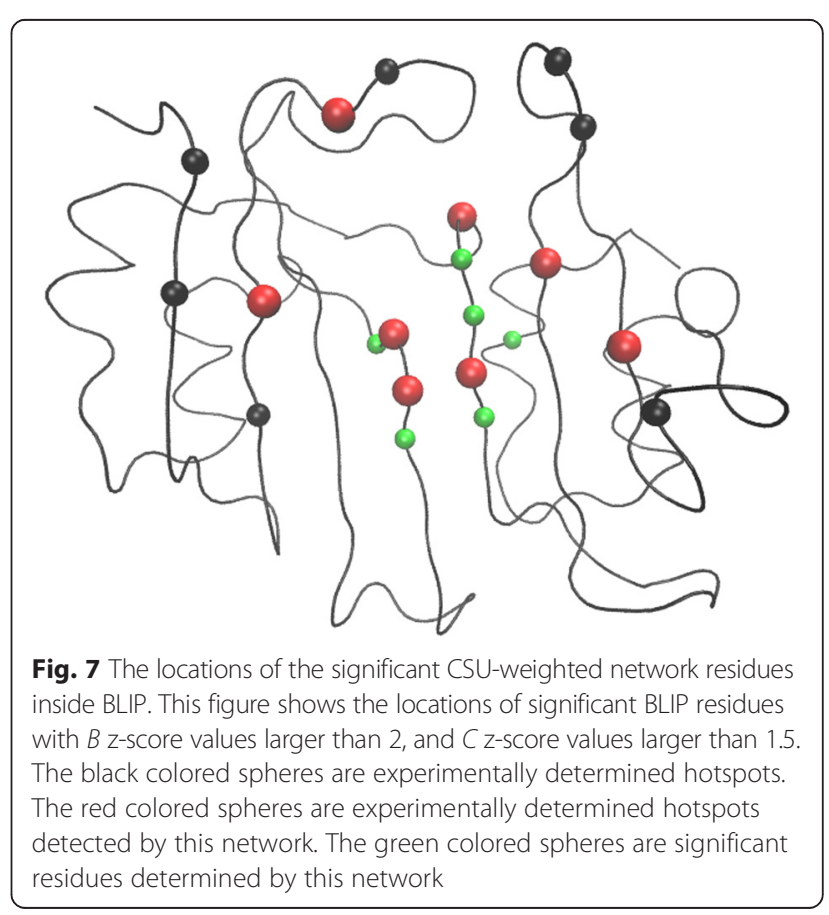




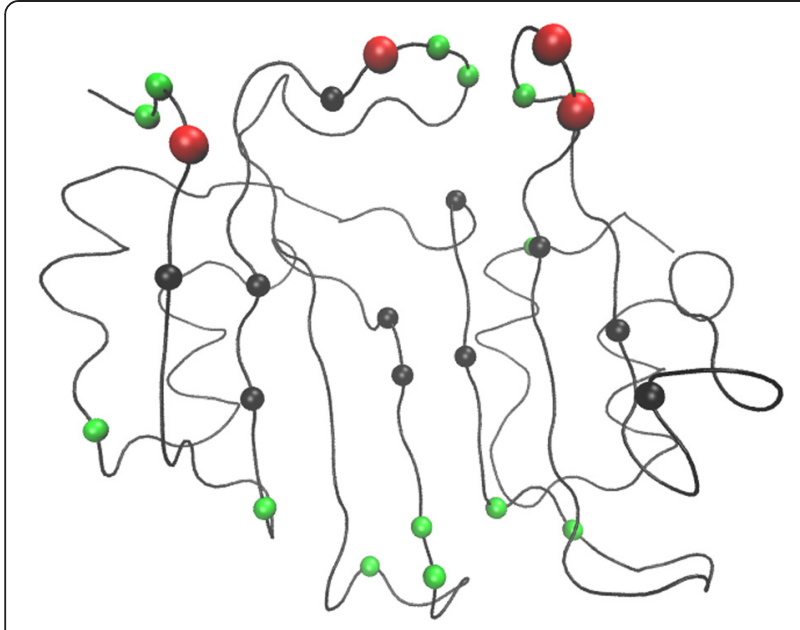

Fig. 8 The locations of the significant JRP-weighted network residues inside BLIP. This figure shows the locations of significant BLIP residues with $B$ z-score values larger than 2 , and $C$ z-score values larger than 1.5. The black colored spheres are experimentally determined hotspots. The red colored spheres are experimentally determined hotspots detected by this network. The green colored spheres are significant residues determined by this network

dimension helps to "unfold" the trajectory. For example, if one inspects a circular orbit along its edge, two points that are not true neighbors could appear to be neighbors due to the low embedding dimension. By using a higher dimension to inspect this circle, in this case two, one can differentiate between true and false neighbors. The embedding dimension chosen would be where the number of false nearest neighbors is very small. The RP then shows the recurrence of a state $X_{i}$ at time $i$ to a former state $X_{j}$ at time $j$ in the phase space trajectory if these two states are within a threshold norm distance $\varepsilon$ from each other. The mathematical expression of the RP matrix is:

$$
R_{i, j}(\varepsilon)=\Theta\left(\varepsilon-\left\|X_{i}-X_{j}\right\|\right) \quad i, j=1, \ldots \ldots \ldots, N
$$

where $N$ is the number of states, $\varepsilon$ is a threshold distance, $\Theta$ is the Heaviside function $(\Theta(x)=0$ if $x<0$ and 1 otherwise), and $\|$.$\| is the Euclidean norm. A recur-$ rence $\left(R_{i j}=1\right)$ in the matrix is represented as a black dot in the plot. The rate of the number of black dots to the total number of points in the matrix gives the recurrence rate value. The Euclidean norm is used due to its intermediate value of neighbors between the maximum and minimum norms. The threshold parameter $\varepsilon$ is the limit that transforms the distance matrix between the states into a recurrence plot of 1's and 0's [33]. A too small $\varepsilon$ would give a small number of recurrences, while a too large $\varepsilon$ would give too many recurrences. This threshold can be defined using different methods [33]. In this work, we chose $\varepsilon$ to give a recurrence rate of $3 \%$. If one is interested in studying how two or more dynamical systems are related to each other, joint recurrences JRP can be used [34], as defined by

$$
\begin{aligned}
J R_{i, j}^{x, y}\left(\varepsilon^{x} \varepsilon^{y}\right) & =\Theta\left(\varepsilon^{x}-\left\|X_{i}-X_{j}\right\|\right) \Theta\left(\varepsilon^{y}-\left\|Y_{i}-Y_{j}\right\|\right), \quad i, j \\
& =1, \ldots N
\end{aligned}
$$

where the JRP has a value of one at time instants when the two systems recur to their simultaneous previous states, and zero otherwise. Equation 4 can be generalized to as many dynamical systems as is needed.

The molecular dynamics simulation and related analysis in this work are performed using the molecular dynamics computer programs NAMD [56] and VMD [57]. The starting BLIP protein structure is downloaded from the protein data bank (PDB: 3gmu) [45]. The simulation details are described elsewhere in detail [28]. The phase space trajectory for each protein residue carbon alpha atom is constructed from a scalar time series of the root mean square deviation RMSD of its position over time [28]. The RMSD values are calculated after removing translational and rotational atomic motions using least square fitting. Each time series consists of 1000 points evenly separated by $10 \mathrm{ps}$, for a total time of $10 \mathrm{~ns}$ for each series. The recurrence parameters of embedding dimension and delay are calculated using the VRA program [58]. The threshold parameter is set to give a recurrence rate of $3 \%$. The recurrence matrices are calculated for all 165 residue carbon alpha atoms using the CRP toolbox routines [59]. By inspecting the recurrence matrices for all 165 residue carbon alpha atoms at all time instants, the number of joint recurrences for each residue pair are accumulated over $10 \mathrm{~ns}$. This number of joint recurrences constitutes the edge weight between the two residues. Each edge weight is consequently divided by the geometric distance between the corresponding residue carbon alpha atoms [6]. For comparison, an un-weighted network with a cutoff threshold distance of $7 \AA$ is prepared. Similarly, a weighted network is prepared with edge weights based on inter-atomic contacts calculated by the CSU program [49]. The $B$ and $C$ values for the three networks are then calculated using the MatlabBGL network toolbox [60]. These values are then standardized by subtracting the mean value, and dividing by the standard deviation for each distribution, respectively. Thus the $B$ and $C$ values for each residue are given as z-scores.

The degree of exposure of each residue to the surrounding water solvent is given by the RSA value for that residue. The RSA values are calculated by dividing the residue's accessible surface area ASA as given by the CSU program [49], by the maximum solvent-accessible surface area of the corresponding standard amino acid residue as calculated recently [61]. Residue with $\mathrm{RSA}<5 \%, \mathrm{RSA} \geq$ 
$5 \%$ and $<20 \%$, and RSA $\geq 20 \%$, are considered buried, partially buried, and exposed, respectively [50].

\section{Abbreviations}

ASA: Accessible surface area; B: betweenness centrality; Bla1: $\beta$-lactamase protein; BLIP: $\beta$-Lactamase Inhibitory Protein; C: Closeness centrality; CPR: Correlation of probability of recurrence; CSU: Contact of structural units; JRP: Joint recurrence plot; NAMD: Not another molecular dynamics; PDB: Protein data bank; RMSD: Root mean square deviation; RP: Recurrence plot; RQA: Recurrence quantification analysis; RSA: Relative solvent accessibility; SHV-1: SME-1, TEM-1: $\beta$-lactamase proteins; VMD: Visual molecular dynamics; VRA: Visual recurrence analysis.

\section{Competing interests}

The authors declare that they have no competing interests.

\section{Authors' contributions}

WIK and NIQ conceived the research idea. NIQ performed all software implementation. WIK analyzed and interpreted the data. WIK prepared the manuscript. Both authors read and approved the final manuscript.

\section{Acknowledgments}

The authors would like to acknowledge the Birzeit University Research Fund (Project \#240168) for supporting this work. The authors would also like to thank anonymous reviewers for constructive comments.

\section{Author details}

'Department of Physics, Birzeit University, Birzeit, Palestine. ${ }^{2}$ Department of Computer Science, Birzeit University, Birzeit, Palestine.

\section{Received: 25 January 2015 Accepted: 18 May 2015}

\section{Published online: 26 May 2015}

\section{References}

1. Csermely P, Korcsmáros T, Kiss HJ, London G, Nussinov R. Structure and dynamics of molecular networks: A novel paradigm of drug discovery: A comprehensive review. Pharmacol Ther. 2013:138(3):333-408.

2. Di Paola L, De Ruvo M, Paci P, Santoni D, Giuliani A. Protein contact networks: an emerging paradigm in chemistry. Chem Rev. 2012;113(3):1598-613.

3. Böde C, Kovács IA, Szalay MS, Palotai R, Korcsmáros T, Csermely P. Network analysis of protein dynamics. Febs Letters. 2007;581(15):2776-82.

4. Taylor NR. Small world network strategies for studying protein structures and binding. Computational and structural biotechnology journal. 2013;5(6). doi:10.5936/csbj.201302006

5. Kannan N, Vishveshwara S. Identification of side-chain clusters in protein structures by a graph spectral method. J Mol Biol. 1999;292(2):441-64.

6. Yan Y, Zhang S, Wu FX. Applications of graph theory in protein structure identification. Proteome Sci. 2011:9 Suppl 1:S17.

7. Sabidussi G. The centrality index of a graph. Psychometrika. 1966;31(4):581-603.

8. Freeman LC. A set of measures of centrality based on betweenness. Sociometry. 1977;35-41.

9. Amitai G, Shemesh A, Sitbon E, Shklar M, Netanely D, Venger I, et al. Network analysis of protein structures identifies functional residues. J Mol Biol. 2004;344(4):1135-46.

10. Del Sol A, Fujihashi H, O'Meara P. Topology of small-world networks of protein-protein complex structures. Bioinformatics. 2005;21(8):1311-5.

11. Bogan $\mathrm{AA}$, Thorn KS. Anatomy of hot spots in protein interfaces. J Mol Biol. 1998;280(1):1-9.

12. De Ruvo M, Giuliani A, Paci P, Santoni D, Di Paola L. Shedding light on protein-ligand binding by graph theory: The topological nature of allostery Biophys Chem. 2012;165:21-9.

13. Vishveshwara S, Brinda KV, Kannan N. Protein structure: insights from graph theory. J Theor Comput Chem. 2002; (01):187-211.

14. VanWart AT, Eargle J, Luthey-Schulten Z, Amaro RE. Exploring residue component contributions to dynamical network models of allostery. J Chem Theory Comput. 2012;8(8):2949-61

15. Ribeiro AA, Ortiz V. Determination of signaling pathways in proteins through network theory: importance of the topology. J Chem Theory Comput. 2014;10(4):1762-9.

16. Vijayabaskar MS, Vishveshwara S. Interaction energy based protein structure networks. Biophys J. 2010;99(11):3704-15.
17. Bizzarri AR, Cannistraro S. Molecular dynamics of water at the protein-solvent interface. J Phys Chem B. 2002;106(26):6617-33.

18. Tarek M, Tobias DJ. Role of protein-water hydrogen bond dynamics in the protein dynamical transition. Phys Rev Lett. 2002:88(13):138101.

19. Fitter J. The Temperature Dependence of Internal Molecular Motions in Hydrated and Dry a-Amylase: The Role of Hydration Water in the Dynamical Transition of Proteins. Biophys J. 1999;76(2):1034-42.

20. Arcangeli C, Bizzarri AR, Cannistraro S. Role of interfacial water in the molecular dynamics-simulated dynamical transition of plastocyanin. Chem Phys Lett. 1998:291(1):7-14

21. Ball P. Water as an active constituent in cell biology. Chem Rev. 2008;108(1):74-108.

22. Zhang L, Yang $Y$, Kao YT, Wang L, Zhong D. Protein hydration dynamics and molecular mechanism of coupled water-protein fluctuations. J Am Chem Soc. 2009;131(30):10677-91.

23. Smolin N, Winter R. Molecular dynamics simulations of staphylococcal nuclease: Properties of water at the protein surface. J Phys Chem B. 2004;108(40):15928-37.

24. Sterpone F, Stirnemann G, Hynes JT, Laage D. Water hydrogen-bond dynamics around amino acids: the key role of hydrophilic hydrogen-bond acceptor groups. J Phys Chem B. 2010;114(5):2083-9.

25. Chakraborty S, Bandyopadhyay S. Correlation between the dynamics of hydrogen bonds and the local density reorganization in the protein hydration layer. J Phys Chem B. 2007;111(26):7626-30.

26. Bagchi B. Water dynamics in the hydration layer around proteins and micelles. Chem Rev. 2005:105(9):3197-219.

27. Bhattacharyya M, Vishveshwara S. Probing the allosteric mechanism in pyrrolysyl-tRNA synthetase using energy-weighted network formalism. Biochemistry. 2011;50(28):6225-36.

28. Fataftah $\mathrm{H}$, Karain W. Detecting protein atom correlations using correlation of probability of recurrence. Proteins: structure, function, and bioinformatics. 2014;82(9):2180-9. doi:10.1002/prot.24574.

29. Eckmann JP, Kamphorst SO, Ruelle D. Recurrence plots of dynamical systems. Europhys Lett. 1987;4(9):973-7.

30. Takens F. Detecting strange attractors in turbulence. In: Dynamical systems and turbulence, Warwick 1980. Berlin Heidelberg: Springer; 1981. p. 366-81.

31. Tsonis AA. Reconstructing dynamics from observables: the issue of the delay parameter revisited. International Journal of Bifurcation and Chaos. 2007:17(12):4229-43

32. Webber Jr CL, Zbilut JP. Dynamical assessment of physiological systems and states using recurrence plot strategies. J Appl Physiol. 1994;76(2):965-73.

33. Marwan N, Carmen Romano M, Thiel M, Kurths J. Recurrence plots for the analysis of complex systems. Phys Rep. 2007;438(5):237-329.

34. Romano MC, Thiel M, Kurths J, von Bloh W. Multivariate recurrence plots. Phys Lett A. 2004;330(3):214-23.

35. Doran JL, Leskiw BK, Aippersbach S, Jensen SE. Isolation and characterization of a beta-lactamase-inhibitory protein from Streptomyces clavuligerus and cloning and analysis of the corresponding gene. J Bacteriol. 1990;172(9):4909-18

36. Strynadka NC, Jensen SE, Johns K, Blanchard H, Page M, Matagne A, et al. Structural and kinetic characterization of a -lactamase-inhibitor protein. Nature. 1994;368(6472):657-9.

37. Strynadka NC, Jensen SE, Alzari PM, James MN. A potent new mode of $\beta$-lactamase inhibition revealed by the $1.7 \AA$ X-ray crystallographic structure of the TEM-1-BLIP complex. Nat Struct Mol Biol. 1996;3(3):290-7.

38. Brown NG, Chow DC, Ruprecht KE, Palzkill T. Identification of the $\beta$-lactamase inhibitor protein-II (BLIP-II) interface residues essential for binding affinity and specificity for class A $\beta$-lactamases. J Biol Chem. 2013;288(24):17156-66.

39. Petrosino J, Rudgers G, Gilbert H, Palzkill T. Contributions of aspartate 49 and phenylalanine 142 residues of a tight binding inhibitory protein of B-lactamases. J Biol Chem. 1999;274(4):2394-400.

40. Zhang Z, Palzkill T. Determinants of binding affinity and specificity for the interaction of TEM- 1 and SME-1 $\beta$-lactamase with $\beta$-lactamase inhibitory protein. J Biol Chem. 2003;278(46):45706-12.

41. Zhang Z, Palzkill T. Dissecting the protein-protein interface between $\beta$-lactamase inhibitory protein and class A $\beta$-lactamases. J Biol Chem. 2004;279(41):42860-6.

42. Reichmann D, Rahat O, Albeck S, Meged R, Dym O, Schreiber G. The modular architecture of protein-protein binding interfaces. Proc Natl Acad Sci U S A. 2005;102(1):57-62.

43. Reichmann D, Cohen M, Abramovich R, Dym O, Lim D, Strynadka NCJ, et al. Binding hot spots in the TEM1-BLIP interface in light of its modular architecture. J Mol Biol. 2007;365(3):663-79. 
44. Wang J, Zhang Z, Palzkill T, Chow DC. Thermodynamic investigation of the role of contact residues of $\beta$-lactamase-inhibitory protein for binding to TEM-1 $\beta$-lactamase. J Biol Chem. 2007;282(24):17676-84.

45. Gretes M, Lim DC, de Castro L, Jensen SE, Kang SG, Lee K, et al. Insights into positive and negative requirements for protein-protein interactions by crystallographic analysis of the $\beta$-lactamase inhibitory proteins BLIP, BLIP-I, and BLP. J Mol Biol. 2009;389(2):289-305.

46. Tuncbag N, Salman FS, Keskin O, Gursoy A. Analysis and network representation of hotspots in protein interfaces using minimum cut trees. Proteins: Structure, Function, and Bioinformatics. 2010;78(10):2283-94

47. Raschke TM. Water structure and interactions with protein surfaces. Curr Opin Struct Biol. 2006;16(2):152-9.

48. Reichmann D, Phillip Y, Carmi A, Schreiber G. On the contribution of water-mediated interactions to protein-complex stability. Biochemistry. 2008;47(3):1051-60.

49. Sobolev V, Sorokine A, Prilusky J, Abola EE, Edelman M. Automated analysis of inter-atomic contacts in proteins. Bioinformatics. 1999;15(4):327-32.

50. Del Sol A, O'Meara P. Small-world network approach to identify key residues in protein-protein interaction. Proteins: Structure, Function, and Bioinformatics. 2005;58(3):672-82.

51. Rajamani D, Thiel S, Vajda S, Camacho CJ. Anchor residues in protein-protein interactions. Proc Natl Acad Sci U S A. 2004:101(31):11287-92.

52. Reynolds KA, Thomson JM, Corbett KD, Bethel CR, Berger JM, Kirsch JF, et al. Structural and Computational Characterization of the SHV-1 $\beta$-Lactamase$\beta$-Lactamase inhibitor protein interface. J Biol Chem. 2006;281(36):26745-53.

53. Reynolds KA, Hanes MS, Thomson JM, Antczak AJ, Berger JM, Bonomo RA, et al. Computational redesign of the SHV-1 $\beta$-lactamase/ $\beta$-lactamase inhibitor protein interface. J Mol Biol. 2008;382(5):1265-75.

54. Grassberger $P$, Schreiber $T$, Schaffrath $C$. Nonlinear time sequence analysis. International Journal of Bifurcation and Chaos. 1991;1(03):521-47.

55. Kennel MB, Brown R, Abarbanel HD. Determining embedding dimension for phase-space reconstruction using a geometrical construction. Phys Rev A. 1992:45(6):3403-11.

56. Phillips JC, Braun R, Wang W, Gumbart J, Tajkhorshid E, Villa E, et al. Scalable molecular dynamics with NAMD. J Comput Chem. 2005;26(16):1781-802.

57. Humphrey W, Dalke A, Schulten K. VMD: visual molecular dynamics. J Mol Graph. 1996:14(1):33-8.

58. VRA. http://softadvice.informer.com/Vra_Eugene_Kononov.html

59. Marwan N. Cross Recurrence Plot Toolbox for Matlab, Reference Manual, Version 5.15, Release 28.6, 2010, http://tocsy.pik-potsdam.de

60. MatlabBGL. https://www.cs.purdue.edu/homes/dgleich/packages/matlab_bgl/

61. Topham CM, Smith JC. Tri-peptide Reference Structures for the Calculation of Relative Solvent Accessible Surface Area in Protein Amino Acid Residues. Comput Biol Chem. 2014;54:33-43. doi:10.1016 / j.compbiolchem.2014.11.007.

\section{Submit your next manuscript to BioMed Central and take full advantage of:}

- Convenient online submission

- Thorough peer review

- No space constraints or color figure charges

- Immediate publication on acceptance

- Inclusion in PubMed, CAS, Scopus and Google Scholar

- Research which is freely available for redistribution

Submit your manuscript at www.biomedcentral.com/submit 